\title{
Surface texturing by solution deposition for omnidirectional antireflection
}

\author{
Meng Tao, ${ }^{\text {a) }}$ Weidong Zhou, ${ }^{\text {b) }}$ Hongjun Yang, and Li Chen \\ Department of Electrical Engineering, University of Texas at Arlington, Arlington, Texas 76019
}

(Received 31 May 2007; accepted 2 August 2007; published online 24 August 2007)

\begin{abstract}
Surface texturing by solution deposition has been developed for antireflection in solar cells. The surface texture is formed by a monolayer of microscale silica particles partially immersed into a spin-on-glass film with a thickness less than the height of the particles. When the silica particles have a spherical shape, the low reflectance from this coating becomes omnidirectional, a desirable feature in fixed-orientation solar panels. It has been experimentally found that the coating improves the transmittance of a quartz wafer in the spectral range of 400-1100 nm and in the incident-angle range of surface normal to at least $30^{\circ}$. The surface texture can be applied to different types of solar cells as an add-on coating. (C) 2007 American Institute of Physics. [DOI: 10.1063/1.2775805]
\end{abstract}

The optical design of a photovoltaic solar cell is to minimize reflection and maximize absorption of incident sunlight. The most successful optical design for commercial solar cells is probably the anisotropically etched pyramids on single-crystalline silicon (100) surface. ${ }^{1,2}$ The microscale pyramids are formed when a flat $\mathrm{Si}(100)$ surface is etched by potassium hydroxide $(\mathrm{KOH})$. With the surface texture, light reflected from a pyramid often strikes another pyramid, thus a second chance of incidence instead of being lost. The record efficiency of $24.4 \%$ in single-crystalline Si solar cells is achieved with this pyramidal surface texture. ${ }^{3}$ The record efficiency of $19.8 \%$ in polycrystalline $\mathrm{Si}$ solar cells is achieved with a lithography-defined surface texture, ${ }^{3}$ since $\mathrm{KOH}$-based anisotropic etching works only on singlecrystalline Si.

For polycrystalline $\mathrm{Si}$ and non-Si solar cells, ${ }^{4}$ there is currently no cost-effective method for surface texturing to minimize reflection. The most-common antireflective coating for these solar cells is a thin film of a transparent conducting oxide (TCO), ${ }^{5}$ although a thin film of $\mathrm{Si}$ nitride is used in polycrystalline $\mathrm{Si}$ solar cells. Indium tin oxide (ITO) has been the most popular TCO. One disadvantage of a thin-film antireflective coating is that reflected light does not get a second chance of incidence. Moreover, thin-film antireflection is typically based on quarterwavelength destructive interference of reflected light. It thus works only in a limited spectral range under near-normal-incidence conditions. Although a laser process has been developed to texture an ITO film, ${ }^{6}$ a more cost-effective and material-independent method for surface texturing is needed.

In this letter, we report surface texturing by coatings from solutions. The surface texture is formed by a monolayer of microscale silica particles partially immersed into a film of spin-on glass (SOG). The solution-based preparation method ensures low cost. The surface texture can be applied to different types of solar cells as an add-on coating. Its low reflectance is a broad spectrum. When the silica particles have a spherical shape, the low reflectance of the surface texture becomes omnidirectional, a desirable feature in solar cells since sunlight strikes the Earth surface from different directions during the day.

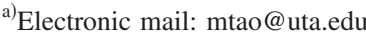

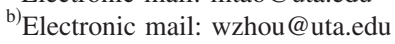

Figure 1 shows how, in principle, a surface texture can be formed by coatings from solutions, in which spherical particles are used as an example. A solar cell surface is first coated with a monolayer of microscale silica particles from a solution containing these particles [Fig. 1(a)]. Then the surface is coated with a film of SOG, which has a refractive index similar to that of the particles [Fig. 1(b)]. The thickness of the SOG film should be a fraction of the diameter of the particles, so the coated surface forms an array of partially spherical particles. When the thickness of the SOG film is the same as the radius of the particles, an array of hemispherical particles is formed. Finally, the coated solar cell is cured to form a spherical surface texture [Fig. 1(c)]. Silica particles of other shapes can also be used, including conical, pyramidal, and tetrahedral. In those cases, the thickness of the SOG film should be a fraction of the height of the particles.

For the results reported in this letter, spherical silica particles of $2 \mu \mathrm{m}$ in diameter from microspheres-nanospheres were used. Monolayer silica particles were spin coated onto both $\mathrm{Si}$ and quartz wafers from a water solution containing $0.1 \mathrm{wt} \%$ of silica particles, i.e., $0.1 \mathrm{~g}$ of particles in $100 \mathrm{ml}$ of water. The coated wafers were heated to $95{ }^{\circ} \mathrm{C}$ in air for 2 min to remove water from the surface. A film of SOG, Honeywell Accuglass $®$ T-11 with a specified thickness range of $0.16-0.26 \mu \mathrm{m}$, was spin coated onto $\mathrm{Si}$ and quartz wafers with monolayer particle coatings. The refractive index of the SOG, 1.39 at $633 \mathrm{~nm}$, is close to that of silica, 1.46, so there is little internal reflection in the coating. Curing for the SOG film was performed first at $80{ }^{\circ} \mathrm{C}$ for $60 \mathrm{~s}$ for solvent removal and then at $130{ }^{\circ} \mathrm{C}$ for $60 \mathrm{~s}$ for cross-linking in the SOG film. The coatings were examined with a Zeiss Supra 55 VP scanning electron microscope (SEM).

Figure 2(a) is a SEM top view of a monolayer of $2 \mu \mathrm{m}$ spherical silica particles coated onto a Si wafer. The particles arrange themselves into multiple domains and within each domain they are close packed. Figure 2(b) is a SEM side

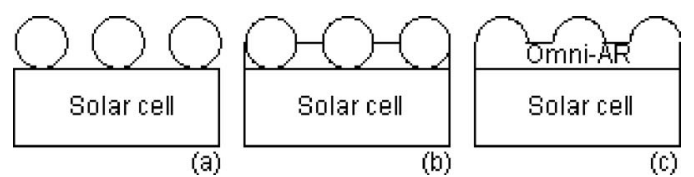

FIG. 1. Schematic of surface texturing by coatings from solutions. (a) Deposition of a monolayer of silica particles, (b) deposition of a spin-on glass film, and (c) curing to form surface texture. 


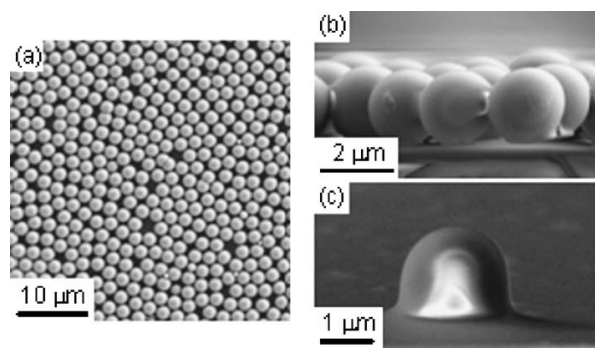

FIG. 2. SEM images of (a) a monolayer of $2 \mu \mathrm{m}$ silica particles coated on a Si wafer (top view). (b) A monolayer of $2 \mu \mathrm{m}$ silica particles coated on a $\mathrm{Si}$ wafer (side view) and (c) a $2 \mu \mathrm{m}$ silica particle immersed in a $0.2 \mu \mathrm{m}$ SOG film.

view of a Si wafer coated with a monolayer of $2 \mu \mathrm{m}$ spherical silica particles. Figure 2(c) shows a $2 \mu \mathrm{m}$ spherical silica particle partially immersed into an $\sim 0.2 \mu \mathrm{m}$ SOG film. Due to capillary effects and high viscosity of the SOG, there is a shoulder region at the base of the partially spherical particle, whose effect needs further investigation.

Since the purpose of an antireflective coating is to maximize light transmission, total transmittance of quartz wafers with various coatings has been measured using a JASCO V-570 spectrophotometer. An integrating sphere was used in the measurement, which collects transmitted light through a sample from all directions. Total transmittance measurements at different incident angles have also been performed using a homebuilt monochromator-based spectroscopic setup with an integrating sphere. The white light from a $100 \mathrm{~W}$ Oriel quartz tungsten halogen lamp was shed on the sample at different incident angles through a liquid light guide. Due to limitations in the setup, the maximum incident angle is limited to $\sim 30^{\circ}$.

Figure 3 shows a normal-incidence total transmittance measurement of a quartz wafer with the described antireflective coating, which comprises a monolayer of $2 \mu \mathrm{m}$ spherical silica particles immersed in a SOG film of $0.2 \mu \mathrm{m}$ thick. For comparison, the total transmittance of a quartz wafer without any coating, a quartz wafer coated with $0.2 \mu \mathrm{m}$ SOG only, and a quartz wafer coated with a monolayer of $2 \mu \mathrm{m}$ spherical silica particles only were also measured. The spherical surface coating improves the transmittance from $\sim 88 \%$ to $\sim 92 \%$ around $400 \mathrm{~nm}$ and from $\sim 90 \%$ to $\sim 92 \%$ around $1100 \mathrm{~nm}$, demonstrating its broad-spectrum antireflection. Since the bare quartz wafer already has a high transmittance above $88 \%$, the improvement by the spherical surface coating is tainted by the high background transmittance.

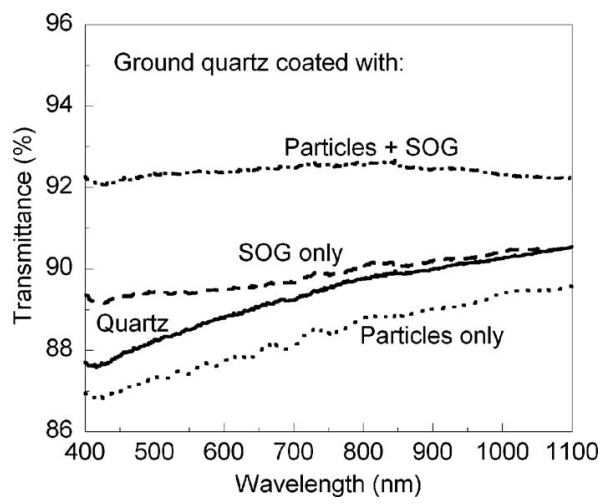

FIG. 3. Total transmittance under normal incidence of quartz wafers with

various surface coatings.
Downloaded 24 Sep 2010 to 129.107 .76 .82 . Redistribution subject to AIP license or copyright; see http://apl.aip.org/about/rights_and_permissions

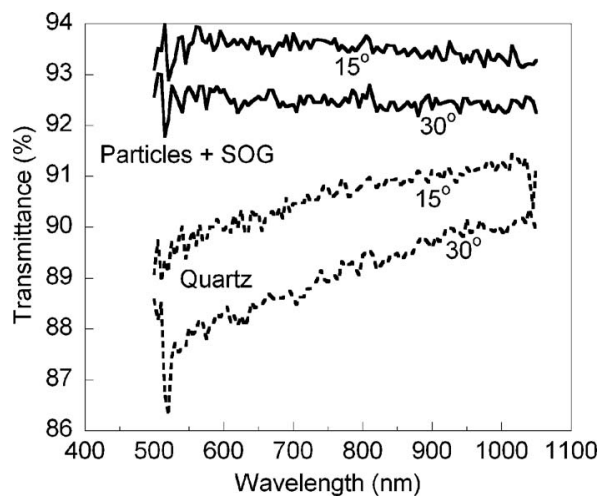

FIG. 4. Angle-dependent transmittance of a quartz wafer before and after a spherical surface coating.

A $0.2 \mu \mathrm{m}$ SOG film alone slightly improves the transmittance at short wavelengths by $\sim 1 \%$, possibly due to its smaller refractive index, 1.39, than silica, 1.46. A monolayer of $2 \mu \mathrm{m}$ silica spherical particles alone decreases the transmittance by $\sim 1 \%$ in the entire spectral range of interest. This is likely due to reflection from the multiple surfaces in the monolayer particle coating: the top surface of the particles, the bottom surface of the particles, and the surface of the quartz wafer. Therefore, the SOG film not only serves as a glue to hold together the coating but also reduces the number of internal surfaces which cause reflection.

A desirable feature of any antireflective coating for solar cells is omnidirectionality, i.e., the antireflection is incidentangle independent. This is particularly important for solar panels with fixed orientations, such as those installed on rooftops and windows. It can also eliminate the need for a mechanical tracking device which ensures normal incidence of sunlight onto a solar panel. Omnidirectionality of the described antireflective coating can easily be accomplished with spherical silica particles partially immersed in a SOG film. Such an array of partially spherical particles keeps incident sunlight at near-normal angles all day long. An incident-angle-dependent transmittance measurement for a spherical surface coating on quartz wafers is shown in Fig. 4. Within the limited range of incident angle $\left(\sim 30^{\circ}\right)$, the spherical surface coating improves the total transmittance from $\sim 87 \%$ to $\sim 92 \%$ around $500 \mathrm{~nm}$ and from $\sim 89 \%$ to $\sim 92 \%$ around $1000 \mathrm{~nm}$ for an incident angle of $30^{\circ}$.

Detailed simulation has been performed using the rigorous coupled-wave analysis, ${ }^{7,8}$ which analyzes the diffraction of an electromagnetic plane wave incident obliquely on a lossless grating structure composed of materials with different refractive indices. All the simulations performed include both transverse-electric (TE) and transverse-magnetic (TM) polarizations. Since we are concerned with nonpolarized sunlight and white light in the measurement setup, the simulated results are simple averages of the calculated TE and TM polarized incident waves. Figure 5 is the simulated transmittance of quartz wafers with and without a spherical surface coating at different incident angles. To match the experimental conditions, the coating in the simulation consists of a $0.2 \mu \mathrm{m}$ SOG film with hemispherical particles of $1 \mu \mathrm{m}$ radius on top. The wavelength-dependent refractive index is assumed to be that of silica and is taken from a book. ${ }^{9}$ The transmittance increases from $\sim 95 \%$ to $\sim 98 \%$ with the described coating at small incident angles of $0^{\circ}$ and $30^{\circ}$. The results agree reasonably well with experiments in license or copyright; see http://apl.aip.org/about/rights_and_permissions 


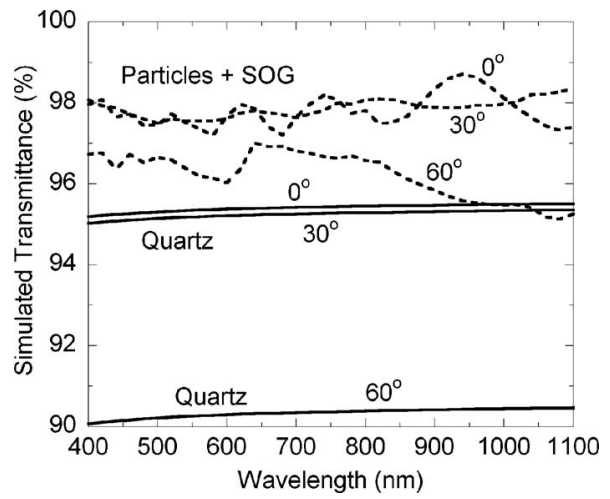

FIG. 5. Simulated transmittance at different incident angles for quartz wafers with and without a spherical surface coating.

Figs. 3 and 4. With a large incident angle of $60^{\circ}$, the transmittance increases from $\sim 90 \%$ to $\sim 96 \%$. This weak dependence of transmittance on incident angle demonstrates omnidirectional antireflection by the spherical surface coating.

The described antireflective coating provides several design parameters to optimize its performance for specific applications. Based on ray optics, ${ }^{10}$ the size of the particles should be greater than the maximum wavelength of interest for the coating to be effective. If a particle size of $1.5 \mu \mathrm{m}$ is chosen, any light with wavelength above $\sim 1.5 \mu \mathrm{m}$ will not be effectively coupled into a solar cell. This means that the infrared portion of the solar spectrum, which heats up a solar cell, will be less effectively coupled into a solar cell than the visible portion by design, leading to a reduced cell temperature. The spherical surface coating is not only good for direct incident sunlight, whose incident angle varies during the day, but also good for diffusive sunlight, which comes in from all directions. This allows effective collection of sunlight under all weather conditions. On a sunny day, the diffusive component of sunlight accounts for $10 \%-20 \%$ of the total solar energy incident on a horizontal surface. On a cloudy day, $100 \%$ of the sunlight is diffusive. ${ }^{11}$ The packing density of the particles is critical for performance. The closest packing of spherical particles of the same diameter is a hexagonal structure. For $2 \mu \mathrm{m}$ spherical particles, this closest packing is equal to $1.92 \times 10^{7}$ particles $/ \mathrm{cm}^{2}$.

In summary, a solution-based method has been developed for surface texturing in solar cells to minimize surface reflection. The coating is formed with a monolayer of silica particles partially immersed in a spin-on glass film with a thickness less than the height of the particles. When the silica particles have a spherical shape, the low reflectance from this coating becomes omnidirectional. It has been experimentally found that the coating improves the transmittance of a quartz wafer in the spectral range of $400-1100 \mathrm{~nm}$ and in the incident-angle range of surface normal to at least $30^{\circ}$. The experimental results are supported by simulation results, which predict angle-independent transmittance up to $60^{\circ}$.

The authors would like to thank K. Han, G. Song, and Z. Qiang for their help with transmittance measurements, and Y. Lvov for attempts to deposit monolayer silica particles by ionic layer-by-layer self-assembly. This work was partially supported by National Science Foundation and Air Force Office of Scientific Research.

${ }^{1}$ R. A. Arndt, J. F. Allison, J. G. Haynos, and A. Meulenberg, Jr., Conference Record of the 11th IEEE Photovoltaic Specialists Conference (IEEE, New York, 1975), p. 40.

${ }^{2}$ D. L. King and M. E. Buck, Conference Record of the 22nd IEEE Photovoltaic Specialists Conference (IEEE, New York, 1991), p. 303.

${ }^{3}$ J. Zhao, A. Wang, M. A. Green, and F. Ferrazza, Appl. Phys. Lett. 73, 1991 (1998).

${ }^{4}$ T. Surek, J. Cryst. Growth 275, 292 (2005).

${ }^{5}$ E. Fortunato, D. Ginley, H. Hosono, and D. C. Paine, MRS Bull. 32, 242 (2007).

${ }^{6}$ H. Hosono, M. Kurita, and H. Kawazoe, Jpn. J. Appl. Phys., Part 2 37, L-1119 (1998).

${ }^{7}$ M. G. Moharam and T. K. Gaylord, J. Opt. Soc. Am. 71, 811 (1981).

${ }^{8}$ K. C. Johnson, Appl. Phys. 24, 249 (1981).

${ }^{9}$ M. J. Weber, CRC Handbook of Laser Science and Technology Supplement 2: Optical Materials (CRC, Boca Raton, FL, 1995), Sec. 2, p. 69.

${ }^{10}$ A. Yariv and P. Yeh, Optical Waves in Crystals: Propagation and Control of Laser Radiation (Wiley, New York, 2002), Chap. 4, p. 79.

${ }^{11} \mathrm{G}$. Smestad, Optoelectronics of Solar Cells (SPIE, Bellingham, WA, 2002), p. 78. 